\title{
Improvement of cancer bleeding in rectal cancer patient with a herbal decoction, Inhyungtang : A case report
}

\author{
Jee Young Lee ${ }^{1}$, SeongHeon Choi ${ }^{1}$, Sora Park ${ }^{2}$, Sung Un Lee ${ }^{2}$, Soomin Lee, \\ Yeehong Jung ${ }^{2}$ \\ ${ }^{1}$ Cancer center of Traditional Korean medicine, Kyung Hee University hospital at Gangdong \\ ${ }^{2}$ Dept. Clinical Korean Medicine, Graduate school of Kyung Hee University
}

\begin{abstract}
Objectives: Rectal cancer bleeding occurs approximately $20 \%$ of overall rectal cancer patients. The current standard treatments cannot cover minor bleeding enough. Failures of the local or systemic approaches to stop bleeding are frequently reported.

Methods: we reported a case of male rectal cancer patient who was treated with herbal decoction and significantly improved on symptoms, such as, loose stools and rectal bleeding. Previous to first visiting to our clinic, he was prescribed to use oral tranexamic acids, which failed to relieve his symptoms. He was administered with an herbal decoction, Inhyungtang, clinically known to have hemostastic effect in hematochezia.

Results \& Conclusion: The prescription showed significant improvement on his symptom, in particular, his bleeding. Thus, we report his case and we expect further experiences and experimental studies to support efficacy of Inhyungtang.
\end{abstract}

$\overline{\text { Key Words }}$ : Inhyungtang, hematochezia, cancer bleeding, colorectal cancer

\section{Introduction}

The UK guidelines characterized the alarm features of colorectal cancer as rectal bleeding, weight loss, change of bowel habit to looser or more frequent stools, a palpable abdominal mass and iron deficiency anemia. ${ }^{1)}$ Although total cancer bleedings occur in approximately $6-10 \%$ of advanced cancer patients, rectal bleeding occurs in about $20 \%$ among rectal cancer patients. Also, compared to other primary cancer lesions, bleeding of rectal cancer can be one of the earliest symptoms and occurs among broad stages. However, rectal bleeding is also regarded as a poor prognostic factor. ${ }^{2-6)}$ Clinicians recommended bleeding control as much as possible, but, not all bleeding can be managed according to the current standard treatment guideline. Sometimes it is such small amount and insignificant to intervene aggressively. Some treatment could be more harmful with counting its side effects. And there are always possibilities of failure.

This is important to treat as clearly as possible because major bleeding may cause death, and minor bleeding may induce anemia that deteriorates the general condition and health-related quality of life, irrespective to underlying diseases even including

\footnotetext{
- Received : 25 November 2014 - Revised : 29 December 2014 - Accepted : 29 December 2014

- Correspondence to : Yeehong Jung

Dept. Clinical Korean Medicine, Graduate School of Kyung Hee University

Cancer Center of Traditional Korean Medicine, Kyung Hee University hospital at Gangdong.

892, Dongnam-ro, Gangdong-gu, Seoul, 134-727, Rep. of KOREA

Tel : +82-2-440-7279, Fax : +82-2-440-7287, E-mail : adagio554@gmail.com
} 
cancer. ${ }^{7)}$ Therefore, all bleeding symptoms should be evaluated and treated but until now only major cancer bleeding was treated locally or systemically. Since current known systemic approach to stop bleeding reported limited efficacy, it becomes standardized to manage locally with the development of endoscopic approach techniques. It is diminished but still exist to fail endoscopic approach such as argon plasma coagulation therapy (APC) or endoscopic submucosal dissection (ESD), ${ }^{2,9,10)}$

Therefore, new systemic therapy from another aspect is required. Since herbal approach to cancer bleeding was rarely reported, here we present a case of Inhyungtang, an herbal decoction clinically known for its hemostatic ability to gastrointestinal, especially to rectum, bleeding on our rectal cancer patient.

\section{Case}

Although this 55-year-old male patient had had loose stools, tenesmus and weight loss for 6 months, he visited the hospital for aggravated hematochezia. Under radiologic evaluation with Computed Tomography (CT) and colonoscopy, he was diagnosed with rectal cancer and mesorectal and pericolic lymphadenopathy but no distant metastasis. (Fig. 1) His initial TNM stage was T3N2bM0 and Duke stage was C. Biopsy confirmed adenocarcinoma, and Epidermal Growth Factor Receptor (EGFR) and K-Ras mutation was not found. Bleeding was not so massive to induce hemodynamic instability but patient complained fatigue and consistent hematochezia. Tranexamic acid was administered to stop bleeding but it didn't work for him.

He moved from previous to our clinic right after his diagnosis and wanted to have both conventional therapy and Traditional Korean Medicine therapy. His initial hemoglobin level was $9.1 \mathrm{~g} / \mathrm{dL}$ and he was hemodynamically stable. He has loose stools more than 15 times a day and bleeding reddened the toilet bowl every time he defecated.

By multidisciplinary diagnosis, he was decided to have preoperative concurrent chemoradiation with capecitabine $825 \mathrm{mg} / \mathrm{m}^{2}$ twice a day with five days a week and sequential transabdominal resection with administration of an additive herbal decoction. We prescribed Inhyungtang decoction, a traditional herbal formula, with oral iron supplements 7 days earlier to
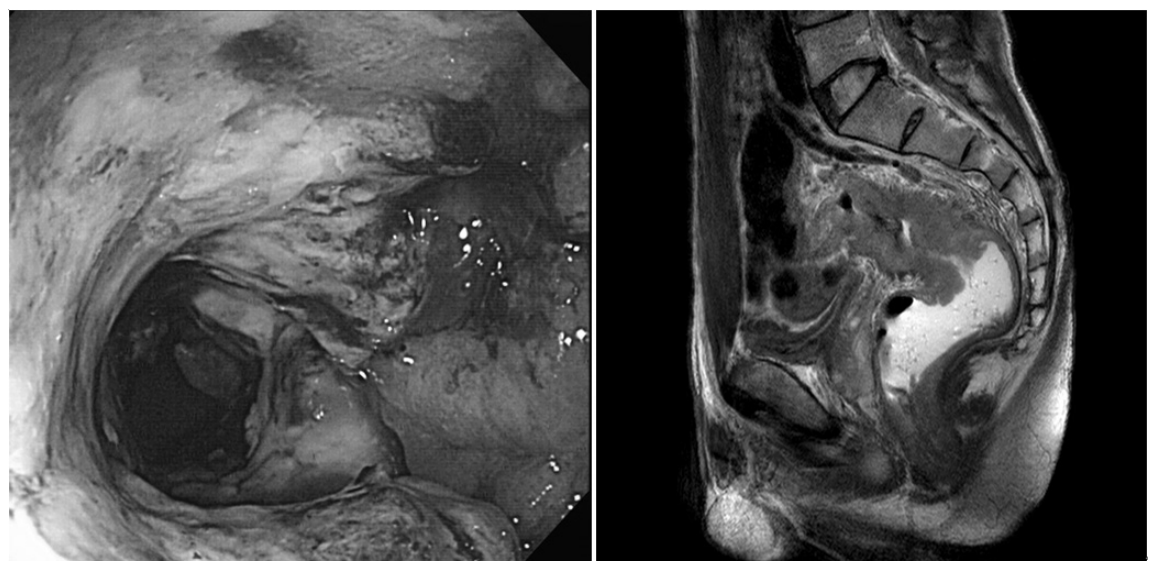

Fig. 1. Colonoscopy and T2-weighted Magnetic resonace imaging (MRI) at his initial work-up (and 6 days before chemoradiation). About $6 \mathrm{~cm}$ of ulcerofungating rectosigmoid colon lesion is observed from $8 \mathrm{~cm}$ to anal verge. 

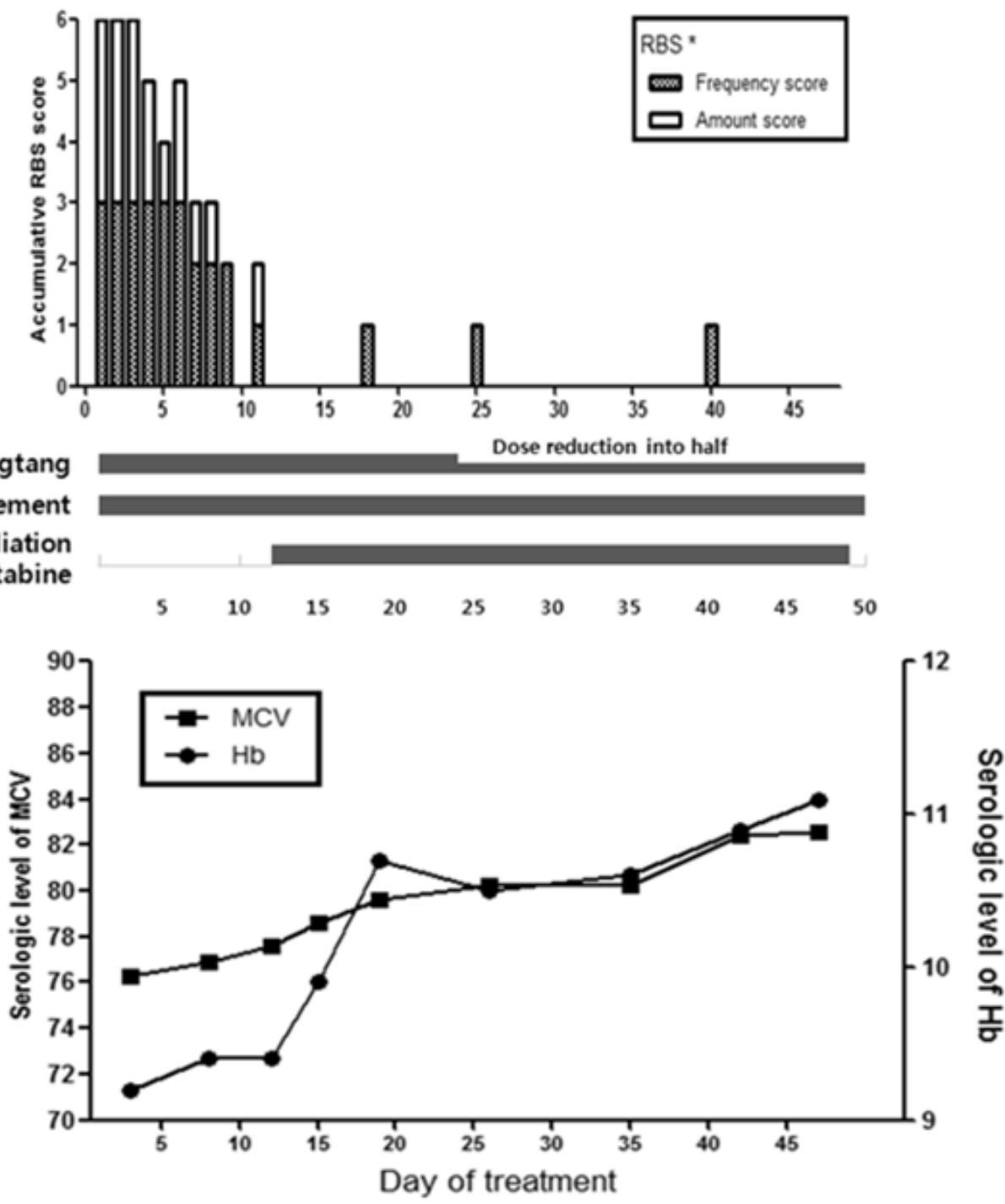

Fig. 2. Treatment schedule and Rectal Bleeding Score (RBS) of the patients (upper) and serologic changes of complete blood cell counts (lower)

* RBS: Scoring system composed of frequency score $(\max 3)$ and amount score $(\max 3)$.

the chemoradiation. (Table 1) Inhyungtang was admi nistered three times a day and his hematochezia showed remarkable improvement during solitary interval of herbal medicine therapy. (Fig. 2) We performed additional acupuncture and moxibustion therapy. Acupuncture therapy was applied at LI4, LI11, ST36, LR3, SP3 to tonify the spleen.
Moxibustion therapy was constantly applied too, at LI11, ST36, BL20 and BL21.

The bleeding was graded with RBS directly and evaluated by complete blood cell count indirectly. (Fig. 2) We evaluated his symptom as Rectal Bleeding Score (RBS). ${ }^{11)}$ This scoring system is derived from Takemoto's study, composed of the 
frequency score and the amount score. The Frequency Score: score 3, 3 or more episodes of bleeding per week; score 2, 0.5-2 episodes per week; and score 1, less than one episode in 2 weeks. The Amount Score: score 3, severe (reddened toilet bowl); score 2, moderate (blood on stool surface); and score 1, mild (blood spot on paper). When bleeding had stopped for over 3 months, RBS was recorded 1.

Table 1. The composition of Inhyungtang

\begin{tabular}{cc}
\hline Scientific name & Amount(g/day) \\
\hline Artemisia capillaries & 8 \\
Alismatis Rhizoma & 8 \\
Poria Cocos & 8 \\
Rhizoma Atractylodis & 4 \\
Polyporus Umbellatus & 4 \\
Typhae Pollen & 3 \\
Schizonepeta Tenuifolia & 3 \\
\hline
\end{tabular}

Due to his physical improvement, dose of the Inhyungtang decoction was reduced into half at the 24th day of the treatment. Moreover, his adverse events of chemoradiation were reported almost nothing. He maintained a little amount of intermittent rectal bleeding for about twice a week and defecation 10 times a day until the time he was under operation. Any bleeding tendency did not appear during and after the operation.

Any adverse event of Inhyungtang was not observed during the treatment.

\section{Discussion}

This rectal cancer patient exhibited almost all of the traditional symptoms followed by his rectal cancer. Of all, his chief complaint was bleeding, which was not improved by tranexamic acid and he was told that the only way to control his bleeding was the shrinkage of his primary tumor. He henceforth visited our clinic for alternative treatment option and experienced improvement with herbal decoction, Inhyungtang.

Bleeding is more frequent symptoms to gastrointestinal cancer than any other cancer. Any colorectal cancer which invades deeper than submucosa can cause bleeding. Approximately 20\% of rectal cancer and even $40 \%$ of colon cancer experienced melena or hematochezia. ${ }^{3)}$ Therefore there are many attempts to control gastrointestinal bleeding but what would fit best to treat rectal bleeding is still controversial.

Clinically significant bleeding requires local control such as packing, dressing, endoscopic coagulation therapy, radiotherapy, and even surgery. Not only local control but systemic intervention, like transfusion, vasopressin, antifibrinolytic agents (e.g. tranexamic acid), also can be applied. ${ }^{8)}$

Endoscopic Argon plasma coagulation (APC) approach showed its efficacy and safety. APC doesn't invade deeper than mucosa, so severe adverse events of APC, including mortality, are much lower than the other local therapies. In fact, Lenz et al said APC would be the best option to stop bleeding effectively. ${ }^{12)}$ But at the same time, limitations of APC are also reported. Tendency of failure of APC to cancer patients is much higher than that of other diseases. There is a report that while $38 \%$ of patients were improved without APC treatment, $31 \%$ of patients were improved with the treatment. ${ }^{13)}$ Recurrence of bleeding after APC was estimated upto $10 \%{ }^{14)}$ Reported APC-related rectal ulcer was in $52-60 \%$ and $10 \%$ of ulcers were symptomatic, requiring additional treatments. ${ }^{11,15)}$

Radiation can be a useful method but when patients have already received maximal dose, it can't be used anymore. ${ }^{2)}$

Hemostatic agents, such as vitamin $\mathrm{K}$, vasoconstrictors, and fibrinolytics, are not ideal either. Vitamin $\mathrm{K}$ may be administered in case of excessive warfarin therapy or liver failure. 
Vasoconstrictor affects myocardial, mesenteric and cerebral circulation and efficacy of vasoactive agents is still controversial. ${ }^{2)}$ Antifibrinolytics showed some efficacy. According to Wardrop et al, there are three considerable randomized controlled trials for antifibrinolytics such as the tranexamic acid and aminocaproic acid. Those trials reported the significant hemostatic efficacy to the bleeding patients including acute leukemia. Platelet transfusions are significantly reduced in the treatment group, and one study found the reduction of red blood cell transfusion out of three. But it is limited because trials were too small and there needs more cancer-related reports. ${ }^{10)}$

There are many herbs and decoctions, decoction methods and various processes of medicinal written in the classics of traditional medicine. Charred formation was known to enhance hemostatic effect. However, only a few of them were proved to be safe and effective. For instance, Yunnan Baiyao, Chinese herbal medicinal powder formulated in Yunnan, was proven to have coagulation effect to the traumatic hemorrhageulcer, and cancer bleeding. Its hemostatic effect on cancer bleeding was presented in series of case reports. ${ }^{16), 17)}$

Formula of Inhyungtang is composed of eight grams of Artemisia capillaries, Alismatis Rhizoma, and Poria Cocos, four grams of Rhizoma Atractylodis and Polyporus Umbellatus, three grams of Typhae Pollen and Schizonepeta Tenuifolia. It comes from Injinsaryeungsan, which is consisted of Alismatis Rhizoma, Poria Cocos, Rhizoma Atractylodis, Polyporus Umbellatus, Artemisia Capillaries, mainly acting to repair damaged liver. ${ }^{18,19)}$ Typhae Pollen and Schizonepeta Tenuifolia were used to reduce heat in the blood. In particular, it is generally known that charred formation of these herbs enhanced hemostatic effect.

Typhae goes into the liver and normalize liver function, particularly prothrombin time $(\mathrm{PT}){ }^{20)}$ It is reported that Typhae also have mild transient vasorelaxation after administration then delayed long and strong vasoconstriction effect. Its effect depends on concentration and Typhae was reported not to damage endothelium. ${ }^{21)}$ There is also an experimental in vivo study of Typhae that significantly reduced PT and activated partial prothrombin time (aPTT) and thereby demonstrated hemostatic properties. ${ }^{22)}$

Schizonepeta was mainly known for anti-inflammatory effect, but it also had hemostatic effect by normalizing aPTT and repairing hematocrit value. ${ }^{23)}$ Both in vitro and in vivo studies of ethanol extracts of charred Schizonepeta proved to have hemostatic effect and when used as charred formation, its hemostatic ability was enhanced. ${ }^{24)}$

This male rectal cancer patient had not experienced any improvement of continuous rectal bleeding with tranexamic acids. He showed reduced amount of bleeding and his laboratory test results (Hemoglobin) got better (9.1 to 11.1) after administration with this herbal decoction, Inhyungtang. Acupuncture to LI4, LI11, ST36, LR3, SP3 and moxibustion to LI11, ST36, BL20 and BL21 was applied to have additional effect to tonify the spleen. None of those were demonstrated to have hemostatic effect by research study. Noticeably, there is no reported adverse event, and no interactions were observed between administration of this herbal medicine and concurrent chemoradiation. In addition, operation schedule was not affected at all by the herbal medicine.

Therefore, we need to be aware of hemostatic efficacy of this herbal decoction, Inhyungtang. It relieved bleeding symptoms without any observed adverse event. Further case reports and fundamental mechanism studies, and some clinical trials are required to confirm its efficacy of Inhyungtang.

\section{Acknowledgements}

We would like to thank our patient, who consented to having his case presented and published. 


\section{Conflict of interest}

The authors declared no conflict of interest.

\section{Reference}

1. Bekkink MO, McCowan C, Falk GA. Diagnostic accuracy systematic review of rectal bleeding in combination with other symptoms, signs and tests in relation to colorectal cancer. $\mathrm{Br} \mathrm{J}$ Cancer. 2010;102(1):48-58.

2. Pereira J, Phan T. Management of bleeding in patients with advanced cancer. The Oncologist. 2004;9(5):561-70.

3. Hamilton W, Round A, Sharp D. Clinical features of colorectal cancer before diagnosis: a population -based case-control study. Br J Cancer. 2005; 93(4):399-405.

4. Rizk SN, Ryan JJ. Clinicopathologic review of 92 cases of colon cancer. S D J Med. 1994;47(3): 89-93.

5. Saidi HS, Karuri D, Nyaim EO. Correlation of clinical data, anatomical site and disease stage in colorectal cancer. East Afr Med J. 2008;85(6): 259-62.

6. Steinberg SM, Barkin JS, Kaplan RS. Prognostic indicators of colon tumors. The Gastrointestinal Tumor Study Group experience.Cancer. 1986; 57(9):1866.

7. Ludwig H, Van Belle S, Barrett-Lee P. The european cancer anaemia survey (ECAS): a large, multinational, prospective survey defining the prevalence, incidence, and treatment of anaemia in cancer patients. Eur J Cancer, 2004;40(15): 2293-306.

8. Laterza L, Cecinato P, Guido A. Management of radiation-induced rectal bleeding. CurrGastroenterolRep. 2013;15(11):355.

9. Jung SJ, Cho Sj, Choi IJ. Argon plasma coagulation is safe and effective for treating smaller gastric lesions with low-grade dysplasia: a comparison with endoscopic submucosal dissection. SurgEndosc. 2013;27(4):1211-8.

10. Wardrop D, Estcourt LJ, Brunskill SJ. Antifibrinolytics (lysine analogues) for the prevention of bleeding in patients with haematological disorders. Cochrane DatabseSyst Rev. 2013;7:CD009733.

11. Chruscielewska-Kiliszek MR, Requla J, Polkowski M. Sucralfate or placebo following argon plasma coagulation for chronic radiation proctitis: a randomized double blind trial. Colorectal Dis. 2012;15(1):e48-55.

12. Lenz L, Tafarel J, Correia L. Comparative study of bipolar electrocoagulation versus argon plasma coagulation for rectal bleeding due to chronic radiation coloproctopathy. Endoscopy. 2011; 43(8):697-701.

13. Takemoto S, Shibamoto Y, Ayakawa A. Treatment and prognosis of patients with late rectal bleeding after intensity-modulated radiation therapy for prostate cancer. RadiatOncol. 2012; 7:87-93.

14. Tomita T, Arai E, Kohno T. Outcomes of treatment of argon plasma coagulation therapy in elderly or high-risk patients with early gastric cancer. J ClinGastroenterol, 2011;45(6):e54-9.

15. Ravizza D, FioriG, Trovato C. Frequency and outcomes of rectal ulcers during argon plasma coagulation for chronic radiation-induced proctitis. GastrointestEndosc. 2003;57(4):519-25.

16. Ladas EJ, KarlikJ, Rooney D. Topical yunnanbaiyao administration as an adjunctive therapy for bleeding complications in adolescents with advanced cancer. Support care cancer. 2012;20(12):3379-83.

17. Yang B, Xu ZQ, Zhang H. The efficacy of Yunnan baiyao on haemostasis and antiulcer: a systematic 
review and meta-analysis of randomized controlled trials. Int J ClinExp Med. 2014; 7(3):461-82.

18. Moon KJ, Yun CW, Lee MJ. The effects of aqua-acupuncture of ArtemisiaeCapillarisHerba extract upon the damaged liver induced by carbon tetrachloride in rats. $\mathrm{J}$ of pharmacopuncture. 1991;8(1):227-39.

19. Ha JY, Baik TH, Jang KS. Effects of Bojungikkitang hap Injinsaryungsan on rat liver injured by CCl4. Korean J Oriental Medical Pathology. 1997;11(1):12-20.

20. Jeong K, AhanBK, Koo BH. An experimental study on the effect to the serum prothrombin time in mouse by Pteropistercus and Tythae Pollen Extract administration. J KH Univ O Med. 1979;2:171-82.
21. Kim RY, Park YS, Kim DI. Vasodilatory activities of the water extracts of seventeen medicinal remedy. J oriental Obstet\&Gynecol. 2005;18(1): 64-80.

22. Ohkura N, Tamjura K, Tanaka A. Experimental study on the hemostatic activity of Pollen Typhae: a traditional folk medicine used by external and oral application. Blood coagul fibrinolysis. 2011;22(8):631-6.

23. Jeong HS, Lee KS, Song BK. Hemostatic effects of Sanguisorbae Radix and NepetaeHerba aqua-acupuncture. J oriental gynecol. 2000;13(1): 18-34.

24. Li O, HuyinC, Peng Z. Research progress on effect of charred Schizonepeta on hamotological system. China Pharmaceuticals. 2010;14: 19-20. 